\title{
Artículo \\ Coronavirus COVID-19: \\ presentación clínica, diagnóstico y tratamiento
}

\section{Coronavirus COVID-19:}

clinical presentation, alagnosis and treatment

\author{
Dra. Cristina Quesada Musa \\ Dra. Michelle Pamela Fung Fallas \\ Dra. Natalia Verónica Medina Correas \\ Médicas generales, egresadas de la Universidad de Costa Rica. \\ Correspondencia: Dra. Natalia Verónica Medina Correas \\ Correo/electrónico: nvmc25@gmail.com
}

\section{Resumen}

Un nuevo coronavirus zoonótico, declarado como pandemia y nombrado como 2019 betacoronavirus novel (2019-nCoV o COVID-19) por la Organización Mundial de la Salud, ha sido identificado como el agente causante de la epidemia de neumonía viral originada en Wuhan, China, a finales del 2019.

Su amplia propagación se debe en gran medida a las características patogénicas que permiten una transmisibilidad eficiente de persona a persona mediante gotas respiratorias, o por el contacto con fluidos corporales; asociada además a la capacidad de contagio por parte de los pacientes asintomáticos. Su diagnóstico se realiza mediante reacción en cadena de polimerasa, en conjunto con la historia clínica, exploración física completa, exámenes de laboratorio y gabinete, para poder clasificar y abordar el caso según su severidad. Actualmente, no se ha desarrollado un 
tratamiento farmacológico específico, eficaz y seguro; el manejo recomendado se basa en controlar la sintomatología y brindar confort al paciente. La prevención incluye precauciones estándar, por gotas y contacto; así como educación a la población para evitar su propagación.

\section{Palabras claves}

Pandemias; infecciones por coronaviridae; betacoronavirus; neumonía viral; transmisión de enfermedad infecciosa; China

\section{Abstract}

A new zoonotic coronavirus, already declared a pandemic and named as a novel betacoronavirus 2019 (2019-nCoV or

COVID-19) by the World Health Organization, has been identified as the causative agent of the viral pneumonia epidemic originated in Wuhan, China, at the end of 2019. Its widespread is largely due to the pathogenic characteristics that allow efficient transmissibility from person to person through respiratory drops, or through contact with body fluids; also associated with the ability to spread by asymptomatic patients. Its diagnosis is made by polymerase chain reaction, together with a medical history, complete physical examination, laboratory and cabinet exams, in order to classify and address the case according to its severity. Currently, no specific, effective and safe drug treatment has been developed; the recommended management is based on controlling the symptoms and providing comfort to the patient. Prevention includes standard precautions, by drops and contact; as well as education to the population to prevent its spread.

\section{Keywords}

Pandemics; coronaviridae infections; betacoronavirus; pneumonia, viral; disease transmission, infectious; China

\section{Introducción}

Los coronavirus corresponden a un tema polémico en la actualidad, siendo el COVID-19 el tercer virus zoonótico en afectar a la población humana en las últimas dos décadas. La cantidad de pacientes infectados por este virus aumenta de manera exponencial conforme avanza el tiempo, a nivel mundial, constituyendo una pandemia que ha generado una respuesta de emergencia. Lo anterior se explica debido a que el virus tiene la característica de ser transmisible de persona a persona y puede ser transmitido por pacientes infectados asintomáticos, siendo el contacto por gotas la principal vía de contagio (1-3).

El abordaje diagnóstico apropiado se basa en datos epidemiológicos, clínicos, radiológicos y de laboratorio. A su vez, la clínica varía dependiendo de las comorbilidades del paciente, pudiendo presentarse asintomático o manifestar síntomas de infección respiratoria severa aguda o shock séptico, en los casos más severos $(4,5)$.

Actualmente, el COVID-19 constituye un desafío para el personal de salud, ya que no existen terapias farmacológicas ni vacunas de eficacia comprobada. Es fundamental adoptar medidas de prevención efectivas tanto de manera intrahospitalaria como en la comunidad, con el fin de controlar la replicación y propagación del virus $(5,6)$.

Este artículo tiene como objetivo reunir y exponer la evidencia reciente sobre el coronavirus, haciendo énfasis en epidemiología, patogenia, abordaje diagnóstico y terapéutico, así como las medidas de prevención, con el fin de brindar herramientas al profesional de la salud para su pronto diagnóstico y tratamiento oportuno en aras de favorecer el pronóstico del paciente. 


\section{Reseña histórica}

Los primeros casos se reportaron a partir del 12 de diciembre del 2019, la Comisión Municipal de Salud de Wuhan reportó 27 casos de neumonía, de los cuales 7 se encontraban críticamente enfermos.

La mayoría de casos presentaban nexo epidemiológico asociado con el mercado de mariscos de Huanan, en la ciudad de Wuhan, donde también se vendían murciélagos, serpientes y otros animales de granja (2). El 7 de enero 2020 la OMS identificó el patógeno como un coronavirus novel el cual fue nombrado provisionalmente como "enfermedad respiratoria aguda COVID-19" por parte del Comité Internacional de Taxonomía de Virus y el 12 de enero se publica la secuencia genómica completa del COVID-19 lo cual permitió un acelerado desarrollo del método diagnóstico por RT-PCR (reacción en cadena de polimerasa con transcriptasa reversa) (1,5,7-11) . El primer caso mortal se reporta el 11 de enero y 2 días después, en Tailandia, es diagnosticado un primer paciente fuera de la zona endémica. $(1,5,7,12)$.

El 20 de enero 2020 se reportaron 15 casos nosocomiales de trabajadores de la salud que habían estado en contacto cercano con pacientes infectados, sugiriendo la transmisión persona a persona y por ende el riesgo de propagación más amplia de la enfermedad $(3,8,13)$. Se declara oficialmente como pandemia el 10 de marzo del 2020 por la OMS. El número de casos confirmados de infección por COVID-19 reportados para el 11 de marzo 2020 llega a cifras de 132758 globalmente, de los cuales 80991 pertenecen a China (3180 muertes) y 51767 son los casos reportados fuera de China, en 122 países (1775 muertes) (14).

El manejo del brote inicial por parte de China fue adecuado al informar oportunamente a la OMS, aislar a pacientes sospechosos de portar el virus, monitorear estrechamente a los contactos, colaborar con la recolección epidemiológica y clínica de los pacientes, y compartir con la comunidad internacional información sobre la secuencia genómica viral $(1,7,13,15)$. La OMS actuó expeditamente al coordinar métodos diagnósticos, instruir en manejo y monitoreo del paciente, recolección de especímenes, tratamiento y proveer información actualizada sobre el brote $(1,5)$. A raíz de esto, múltiples países iniciaron un tamizaje a viajeros provenientes de Wuhan que presentaran cuadro febril o nexo epidemiológico positivo, con el objetivo de detectar el COVID-19 e intentar evitar que el mismo llegase a convertirse en pandemia $(5,15,16)$

\section{Metodología}

Para la elaboración de este artículo, se utilizaron las bases de datos de PubMed, Clinical Key, Science Direct y JAMA Network para buscar bibliografía no mayor a 3 meses de antigüedad (desde enero hasta marzo del año 2020). Se utilizaron los términos "coronavirus", "novel coronavirus", "infección", "2019-nCoV" y "COVID-19", combinados con "clínica",

"fisiopatología", "diagnóstico" y "tratamiento". La búsqueda se enfocó en la información más actualizada y en los artículos que tuvieran mayor relevancia y vigencia con el contenido a exponer en este trabajo

\section{Epidemiología}

Se identificaron los siguientes hallazgos epidemiológicos relacionados con el COVID-19 la mayoría de pacientes infectados son hombres (64-73\%, con un radio de $2-7$ casos presentados en hombres por cada caso en mujeres), esta susceptibilidad disminuida en las mujeres puede ser atribuible a la protección que brinda el cromosoma X y las hormonas sexuales que juegan un rol importante en el sistema inmune (17). La edad media fue de 49 años (sin embargo cualquier grupo etario puede ser afectado), al 
inicio del brote aproximadamente $50 \%$ de los pacientes habían tenido contacto directo con el mercado de mariscos Huanan, alrededor de la mitad de los pacientes presentaron enfermedades crónicas subyacentes y cerca del $15 \%$ falleció $(1,2,8,12,17)$.

A pesar de los datos anteriores, se sospechaba que el riesgo de mortalidad de este agente viral era mucho menor al obtenido inicialmente, posiblemente por el sesgo generado por casos de mayor severidad. Dicho esto, en marzo del 2020 con 132758 casos confirmados, la tasa de mortalidad asociada a esta neumonía viral presenta cifras que rondan el $3,7 \%$, tanto en China como en otros 122 países. Adicionalmente, la mayoría de pacientes que fallecieron a causa de este agente viral presentaban compromiso de su inmunidad por condiciones médicas subyacentes como hipertensión, diabetes o enfermedades cardiovasculares $(1,5,8,14,17,18)$. A modo de resumen, el mayor riesgo de contagio del virus COVID-19 se asocia a masculinos mayores de 40 años, con riesgo de mortalidad incrementado para individuos con comorbilidades crónicas.

\section{Patogénesis}

Los coronavirus corresponden a un grupo de virus que causan un porcentaje significativo de resfriados humanos comunes, tanto en adultos como en niños inmunocompetentes. Los coronavirus $(\mathrm{CoVs})$, son virus ARN envueltos, de una sola cadena en sentido positivo, de origen zoonótico y pertenecientes a la familia Coronaviridae del género beta coronavirus, subgénero de Sarbecovirus, del orden Nidovirales $(2,7,16,19)$.

Los CoVs emergentes, incluyen el síndrome respiratorio agudo severo coronavirus (SARS-CoV) que causa infecciones en vía respiratoria superior e inferior, el síndrome respiratorio del medio oriente coronavirus (MERS-CoV), y el COVID-19 siendo el sétimo coronavirus que ha infectado a la población humana, y el tercero en las últimas dos décadas; estos tres pertenecientes a los beta coronavirus, han generado un estado de alerta para las instituciones públicas mundiales. Hasta el momento el COVID-19 ha exhibido un comportamiento menos patogénico que el SARS-CoV y en mucha menor proporción que el MERS-nCoV. Al comparar el índice de mortalidad del COVID-19 (o 2019-nCoV) con enfermedades virales previas, obtenemos los siguientes datos: COVID-19 menos del 4\%, H1N1 0.02-0.04 \%, H7N9 39\%, SARS-CoV 9.5\%, MERS-CoV 34.4\% y Ébola 63\%; de estas son pandémicas el SARS, H1N1 y recientemente el COVID-19 $(1,2,8,11,12,15,20)$.

La secuencia genómica del COVID-19 es similar en un $89 \%$ al SARS-CoV y en un $82 \%$ al tipo SARS de los murciélagos. Se ha reportado que el COVID-19 utiliza el mismo receptor de entrada celular que el SARS-CoV, esta proteína receptora llamada ACE2 se encuentra en gran cantidad en humanos en el epitelio pulmonar y de intestino delgado, al igual que los coronavirus también se encuentran en tracto gastrointestinal y vía respiratoria superior de los mamíferos. De esta manera, el identificar la posible ruta de infección puede ayudar a dilucidar la patogénesis y tratamiento de la enfermedad causada por el COVID-19 $(1,5)$.

La fuente de origen de este virus zoonótico se determinó como proveniente de los murciélagos, siendo su reservorio más probable las serpientes $(2,12,21)$. No obstante, se han generado una cantidad creciente de casos debido a la transmisión por contacto entre interpersonal. Según un análisis realizado por la Universidad de Georgetown, se cree que el periodo de incubación es de aproximadamente 14 días, por lo que se presume la primoinfección tomó lugar en noviembre 2019. Esto resultó en la declaración de esta pandemia como una emergencia de salud pública de Interés internacional, por parte de la OMS $(6,18,22-24)$. 


\section{Transmisibilidad}

\section{Transmisibilidad persona a persona}

Uno de los pasos clave para la transmisibilidad de un virus es el salto de los animales a los humanos. En este caso, los orígenes de dicho paso remontan al mercado mayorista de mariscos en Wuhan, China, donde también se vendían animales vivos de manera ilegal. Esto debido a que la mayoría de los primeros pacientes tenían un historial reciente de exposición a animales salvajes en dicho mercado, por lo que se cree que estas personas fueron infectadas por exposiciones zoonóticas o ambientales $(2,11,12,19,25)$.

Actualmente se confirmó la transmisión eficiente de persona a persona por parte de este virus, lo cual ha influenciado su propagación a mayor escala. Lo mismo se constató mediante casos confirmados del virus en un grupo familiar que carecía de antecedentes conocidos de contacto con animales, visitas a mercados o ingesta de carne de animales de caza en restaurantes, pero tenían historial positivo de contacto con personas hospitalizadas por neumonía recientemente $(5,10,21,26,27)$; así como por infecciones documentadas en el personal de salud que atendía a pacientes con COVID-19, pacientes hospitalizados en las mismas salas e informes de familias y viajeros infectados al provenir de ciertas regiones geográficas, sin antecedente de haber asistido a mercados o haber tenido contacto con animales $(5,10,12,13)$.

El COVID-19 se transmite principalmente mediante gotas respiratorias de más de 5 micras, al toser, estornudar o hablar. También por contacto directo con fluidos corporales de pacientes infectados o contacto con superficies y animales contaminados $(3,5)$. Asimismo, existe la posibilidad de contagio por aerosoles en procedimientos terapéuticos que los produzcan (9).

Por otro lado, al tratarse la presente enfermedad de un virus, su transmisibilidad puede cambiar estacionalmente según el clima o las reuniones anuales, donde hay mayor acercamiento de personas (5).

\section{.Período de incubación}

La rápida propagación del COVID-19 se asocia también a un largo período de incubación, el cual es en promedio de 5.2 días (y hasta 12.5 días, según diversos estudios) (25); período en el cual personas asintomáticas son potenciales fuentes de infección (24). Además, se ha observado que en sus estadios tempranos, la epidemia duplica su tamaño cada 7.4 días; y que los pacientes subclínicamente sintomáticos pueden no solo transmitir el virus al inicio de su enfermedad, sino también eliminar grandes cantidades del mismo e infectar a otros incluso después de recuperarse de la enfermedad aguda $(3,25,28)$. Es por lo anterior que la gravedad de la enfermedad es un factor indirecto importante en la capacidad de propagación del virus, ya que si la infección no causa una enfermedad grave, las personas infectadas probablemente no busquen atención médica y propaguen el virus a sus contactos $(15,25,29)$.

\section{Diagnóstico \\ Clínico}

De acuerdo a las guías de la OMS del 2020 sobre el coronavirus COVID-19 se debe realizar una amplía evaluación de la historia clínica y sospechar la infección en los grupos de pacientes que se muestran a continuación: $(5,18,25)$

- Pacientes con historia de infección respiratoria severa aguda (fiebre, tos $\mathrm{y}$ hospitalización reciente por esta causa) y sin ninguna etiología que explique la presentación clínica. Adicionalmente que el paciente remita historia de viaje reciente (menos de 14 días), resida en Wuhan, China o sea un trabajador de la salud con infecciones respiratorias agudas severas sin etiología clara.

- Pacientes con cualquier patología respiratoria aguda que posea síntomas en un periodo máximo de 14 días posterior a la 
exposición y presente alguno de los siguientes contactos previos: contacto cercano (exposición laboral o convivencia diaria con algún paciente infectado), contacto por visita o trabajo en un mercado animal en China y, por último, persona que labora o atiende en un área de salud con casos confirmados de infección.

El coronavirus se presenta principalmente en hombres mayores de 40 años y gran parte de los casos sintomáticos presentan neumonía viral como parte de su presentación clínica. La fase prodrómica asociada al coronavirus COVID-19 incluye sintomatología inespecífica: fiebre, mialgias, tos seca, disnea y fatiga $(5,8,25)$. Los pacientes asocian también síntomas menos comunes como cefalea, expectoración, mareos, dolor abdominal, náuseas, vómitos, hemoptisis y diarrea. Dentro de las complicaciones más comunes se encuentran el síndrome de distrés respiratorio agudo, seguido por lesión cardiaca aguda e infecciones secundarias $(8,12)$.

$\mathrm{Al}$ examen físico se debe verificar la existencia de inestabilidad hemodinámica, lo cual es sugestivo de sepsis o shock séptico, por lo cual la monitorización de los signos vitales es primordial en casos sospechosos de infección por coronavirus. A la exploración física es necesario buscar datos sugestivos de dificultad respiratoria tales como utilización de músculos accesorios, aleteo nasal, crépitos a la auscultación o murmullo vesicular disminuido $(8,25)$.

Según las guías de la OMS 2020 la infección por coronavirus COVID-19 puede asociar 6 síndromes clínicos: enfermedad no complicada, neumonía leve, neumonía severa, distrés respiratorio agudo, sepsis y shock séptico. Lo anterior comprende los grupos etarios de adultos y niños menores a 2 meses hasta 5 años, sin embargo, para efectos del artículo se enfatizará en el grupo etario adulto. Los pacientes con enfermedad no complicada presentan síntomas inespecíficos (fiebre, tos, faringodinia, congestión nasal, malestar general, cefalea o mialgia) y se debe monitorizar a los adultos mayores e inmunosuprimidos por síntomas atípicos; este grupo no incluye manifestaciones como deshidratación, sepsis o disnea (18). Los dos parámetros clínicos primordiales a evaluar en los pacientes con neumonía son la frecuencia respiratoria y la saturación de oxígeno. Para sepsis en adultos se evalúan principalmente el estado mental del paciente, la circulación y los parámetros respiratorios. Por último, en shock séptico se debe valorar la hipotensión o niveles de lactato mayores a $2 \mathrm{mmol} / \mathrm{L}(2,25)$.

Los criterios de alta hospitalaria incluyen cese de la fiebre por al menos 10 días, con mejoría de hallazgos en radiografía de tórax y cultivos respiratorios negativos por virulencia. Cabe destacar que la mayoría de los pacientes fallecidos debido al coronavirus presentaban inmunocompromiso asociado a comorbilidad con hipertensión arterial, diabetes mellitus o enfermedad cardiovascular principalmente, además de neoplasias, enfermedad cerebrovascular, enfermedad renal o hepática crónica e infección por VIH $(1,5,8,15)$.

El COVID-19 debe diferenciarse de otras cepas de la familia coronaviridae, ya que en el pasado se han mostrado casos asociados a MERS-CoV (Síndrome respiratorio del Medio Este) y SARS-CoV. Las manifestaciones clínicas del MERS-CoV en el brote del 2012 incluía un grupo etario similar con mayor sintomatología de disnea, diarrea, faringodinia y en general se requería mucho mayor soporte ventilatorio. El SARS-CoV era menos disneizante por lo que la necesidad de soporte ventilatorio era menor y presentó una tasa de mortalidad inferior al MERS-CoV.

El COVID-19 no presenta faringodinia con bajo requerimiento de soporte ventilatorio según datos reportados a la fecha. Realizar diagnóstico diferencial con otras entidades tales como virus respiratorio sincitial en niños, virus de la 
influenza y neumonías adquiridas en la comunidad (tanto bacterianas como virales) resulta de vital importancia $(5,16)$.

\section{Pruebas de laboratorio}

El gold estándar para el diagnóstico de la infección COVID-19 es la detección de ácidos nucleicos virales por RT-PCR (reacción en cadena de polimerasa con transcriptasa reversa) en muestras de vías respiratorias superiores mediante hisopado nasofaríngeo y orofaríngeo, se recomienda incluir también muestras de tracto respiratorio inferior como esputo o secreciones bronquiales. La recolección de muestras se debe llevar a cabo con hisopos de dacrón estéril (no utilizar algodón) evitando la toma de muestras de fosas nasales o amígdalas (18). Una muestra del tracto respiratorio superior negativa no excluye el diagnóstico y se recomienda realizar en conjunto con muestras de tracto respiratorio inferior. En pacientes hospitalizados es necesario repetir la toma de ambas muestras al menos cada 2 a 4 días para demostrar el aclaramiento del virus $(4,7,11)$.

Es recomendable la recolección de hemocultivos asociados a neumonía y sepsis, idealmente antes de implementar una terapia antimicrobiana. Adicionalmente el abordaje diagnóstico incluye la realización de un hemograma completo, pruebas de función hepática, pruebas de coagulación y reactantes de fase aguda; los hallazgos más comunes comprenden leucocitosis con neutrofilia, anemia, linfopenia y trombocitopenia, disminución del tiempo de protrombina e incremento en ALT y AST. En los reactantes de fase aguda la procalcitonina se mantiene dentro de rangos normales, sin embargo la proteína $\mathrm{C}$ reactiva (PCR) y la velocidad de eritrosedimentación (VES) aumentan $(12,13,17,19)$.

Existen otros hallazgos de laboratorio más específicos como aumento de dímero D, troponina I, IL1B, IL1RA, IL2, IL7, IL8, IL9, IL10, factor de crecimiento de fibroblastos (FCF) e IFNY $(8,17)$.

\section{Técnicas de imagen}

El abordaje diagnóstico debe incluir una radiografía de tórax, la cual puede tener cambios asociados a una neumonía como infiltrados lobulares, consolidaciones bilaterales o unilaterales (es menos común), patrón en vidrio esmerilado $\mathrm{y}$ en pocas ocasiones puede mostrar neumotórax $(10,17,19)$.

La tomografía axial computarizada no contrastada (TAC sin contraste) de pacientes infectados con coronavirus aporta mayor relevancia diagnóstica con hallazgos que pueden incluir consolidaciones múltiples, bilaterales, subsegmentarias o lobulares; las lesiones presentan una distribución subpleural predominantemente. Tardíamente los pacientes con neumonía por coronavirus mostraron en imágenes de TAC sin contraste opacidades bilaterales de predominio basal en patrón de vidrio esmerilado con resolución de las áreas de consolidación, lo cual es indicativo de progresión de la enfermedad $(4,8,30)$

\section{Tratamiento}

De acuerdo con las guías de la OMS, no se cuenta con un tratamiento específico confirmado que pueda ser utilizado de manera rutinaria en estos casos. El manejo actual se basa en medidas de soporte que controlen la sintomatología y brinden confort al paciente, además de antibioticoterapia empírica $(2,18)$.

\section{Medidas de soporte}

- Oxigenoterapia: Se debe iniciar tratamiento suplementario con oxigenoterapia a una dosis de $5 \mathrm{~L} /$ minuto en pacientes infectados con enfermedad respiratoria severa aguda y que presenten distrés respiratorio, hipoxemia o shock. La meta de saturación de oxígeno es mayor o igual a $90 \%$ en adultos y mayor a $92-95 \%$ en embarazadas. Se recomienda utilizar ventilación mecánica en casos graves y potencialmente mortales $(2,18)$. 
- Fluidoterapia: El manejo de la fluidoterapia en pacientes con enfermedad respiratoria severa debe ser conservador si no hay evidencia de shock subyacente, debido a que una resucitación agresiva puede empeorar la oxigenación, especialmente en escenarios donde existe limitada disponibilidad de ventilación mecánica $(2,18)$.

- Analgesia: En caso de ser necesario (2).

\section{Terapia antimicrobiana}

A pesar de que se sospeche que el paciente se encuentra infectado con COVID-19, es recomendado administrar terapia empírica apropiada con antibióticos durante la primera hora de identificación de sepsis. El tratamiento antimicrobiano se debe basar en el diagnóstico clínico (neumonía adquirida en la comunidad u hospitalaria, o sepsis), epidemiología local y comorbilidades. Se debe desescalonar la terapia cuando existen resultados microbiológicos negativos por bacterias o confirmación de infección viral. Por otro lado, las guías chinas recomiendan su uso sólo cuando existe evidencia de infección bacteriana $(2,18)$.

\section{Esteroides}

En las guías chinas para el manejo del COVID-19 se recomienda su uso para reducir la autolesión generada por la activación del sistema inmune. Sin embargo, la OMS no recomienda el uso rutinario de esteroides sistémicos en el tratamiento de neumonía viral, salvo que esté indicado. Lo anterior se basa en estudios observacionales de pacientes con neumonía aguda severa de etiología viral donde la administración de corticosteroides no mostró beneficio en cuanto a supervivencia y aumentó los eventos adversos (infecciones secundarias, necrosis avascular, psicosis, diabetes $\mathrm{y}$ aclaramiento viral retrasado) $(2,18)$.

Además se han estudiado los siguientes tratamientos complementarios:

\section{Tratamiento general para infección viral}

- Intervenciones nutricionales: Liu estudió que la respuesta inmune a menudo se ha visto debilitada por una nutrición inadecuada, por lo que propone la ingesta de suplementos nutricionales como opciones complementarias al tratamiento farmacológico, entre ellos: vitamina $A$, vitamina $B$, vitamina $C$, vitamina $\mathrm{D}$, vitamina $\mathrm{E}$, ácidos grasos poliinsaturados (omega-3 y omega-6), selenio, zinc y hierro (6).

- Inmunomoduladores: Al igual que el soporte nutricional, diversos estudios sugieren que pueden ser utilizados como tratamiento coadyuvante del COVID-19 para mejorar la inmunidad del huésped contra el virus. Dentro de las opciones se encuentran: interferones (IFNs), gammaglobulinas intravenosas (IVIg), timopentina (TP5), levamisol, derivados no inmunosupresores de ciclosporina A y medicina china (glicirricina, baicaleína y ginseng).

Además, se recomienda utilizar timosina alfa-1 antes de la administración de metilprednisolona, ya que aumenta la resistencia a la apoptosis de timocitos inducida por los glucocorticoides (6).

\section{Tratamiento específico para coronavirus}

- Inhibidores de la proteasa del coronavirus: Constituyen los inhibidores de quimotripsina como la cinanserina y los flavonoides, e inhibidores de la proteasa similar a la papaína como los diarilheptanoides. La quimiotripsina y la proteasa similar a la papaína son esenciales para la replicación del coronavirus y la inhibición de la respuesta inmune innata del huésped, por lo que al inhibirlas se disminuyen estas funciones (6). 


\section{Prevención}

- Bloqueadores de la unión de la enzima convertidora de angiotensina 2 o inhibidores de la proteína S: Anticuerpo monoclonal humano (mAb), emodina, promazina y nicotianamina. Disminuyen la infección por coronavirus al interferir con la ECA2, uno de los sitios de unión del coronavirus (compiten en el sitio de unión o lo bloquean) $(6,7)$.

- Tratamientos antivirales: Ribavirina, lopinavir + ritonavir, oseltamivir, ganciclovir, remdesivir. Cabe destacar que el remdesivir es un análogo de adenosina que se incorpora entre las cadenas de ARN y resulta en terminación prematura, funcionando en un estado posterior a la entrada del virus (31). Los estudios revelan que podría considerarse el uso de antivirales en combinación con otros tratamientos para reducir la tasa de reproducción viral, principalmente al inicio de la enfermedad; sin embargo aún se necesita comprobar su eficacia y seguridad $(2,6,10,18)$.

- Antimaláricos: Las investigaciones actuales han centrado su atención en la cloroquina, la cual presenta acción antiviral e inmunomoduladora que actúan en sinergia.

En cuanto a su mecanismo de acción antiviral, la cloroquina aumenta el $\mathrm{pH}$ endosomal que se requiere para la fusión del virus a la célula, actuando tanto a nivel de entrada viral como posterior a la misma. Un estudio realizado en China en febrero del 2020 reportó la eficacia de la cloroquina en la infección por COVID-19 y plantea que se incluirá dentro de la próxima edición de las guías chinas, sin embargo, su uso no se encuentra protocolizado a la fecha $(31,32)$.

- Otros compuestos: Ácido alfa lipoico, estradiol y fitoestrógeno, mucroporin-M1 (6).
Las medidas de prevención y control de la infección son una parte fundamental e integral del manejo y deben iniciarse desde el ingreso del paciente al hospital. De acuerdo a la guías de la OMS, las precauciones adecuadas para el manejo de los pacientes en investigación o confirmados por infección de COVID-19 incluyen: precauciones estándar, precauciones de transmisión por gotas y precauciones por contacto $(8,10,18)$.

\section{Precauciones estándar: $(13,18)$}

- En el triaje dar al paciente sospechoso una mascarilla y dirigirlo a una sala de aislamiento.

- Higiene de manos, especialmente después del contacto con secreciones respiratorias.

- Uso de equipo personal protector (mascarilla, protección para ojos, guantes y bata) para evitar contacto directo con sangre, fluidos corporales, secreciones y piel no intacta de los pacientes.

- Prevención de lesiones por objetos punzocortantes.

- Eliminación segura de residuos.

- Limpieza y desinfección de equipos.

- Limpieza del medio ambiente.

\section{Precauciones de transmisión por gotas: $(8,18)$}

- Lavado de manos.

- Cubrirse la boca y nariz al toser y estornudar.

- Gafas y mascarilla quirúrgica si se trabaja a 1-2 metros del paciente.

- Colocar a los pacientes en habitaciones 
individuales o agrupar a aquellos con el mismo diagnóstico etiológico o con diagnóstico clínico similar + factores de riesgo epidemiológico.

- Limitar el movimiento del paciente dentro de la institución.

\section{Precauciones de transmisión por contacto: $(3,18)$}

- Uso de equipo personal protector al entrar a la habitación y desecharlo al salir.

- Limpiar y desinfectar el equipo compartido entre pacientes.

- Evitar tocarse los ojos, nariz y boca con las manos enguantadas o sin guantes potencialmente contaminadas.

- Evitar contaminar superficies ambientales que no están directamente relacionadas con el cuidado del paciente (por ejemplo, manijas de puertas e interruptores de luz).

- Asegurar una ventilación adecuada de la habitación.

- Evitar el movimiento de pacientes.

- Higiene de manos.

\section{Precauciones de transmisión por generación de aerosoles: $(3,18)$}

En caso de succión abierta del tracto respiratorio, intubación, broncoscopía o reanimación cardiopulmonar.

- Mascarilla de alta eficacia (N95 o equivalente, o mayor nivel de protección).

- Gafas.
- Guantes.

- Batas impermeables de manga larga.

- Evitar la presencia de personas innecesarias en la habitación.

\section{Otras medidas:}

- Campañas educativas: Promover precauciones para los viajeros, incluyendo lavarse las manos con frecuencia y usar mascarillas al visitar lugares públicos; así como restricción de viajes a zonas con casos sospechosos o confirmados. Educar al público sobre la higiene personal y alimentaria $(2,5,12,13)$.

- Obtener información por parte del personal de salud: Historial reciente de viajes o exposición a contactos enfermos en cualquier paciente que se presente a recibir atención médica con síntomas de enfermedad aguda, con el fin de garantizar la identificación adecuada y el aislamiento inmediato de pacientes que pueden estar en riesgo de infección por COVID-19 y así ayudar a reducir la transmisión adicional (10). Además es importante mantener una constante vigilancia sindrómica (fiebre) y rastreo activo de contactos $(5,15)$.

- Vacunación: Actualmente, no existe una vacuna efectiva contra este virus. La aprobación de vacunas para prevenir la infección por el COVID-19 se ha retrasado debido a la falta de modelos animales adecuados para evaluar su eficacia, conocimiento de mutaciones de las proteínas objetivo, asentimiento de que las vacunas de ADN pueden recombinarse con otros virus y de que la inmunidad preexistente puede eliminar la vacuna al eliminar los vectores generales del virus humano, además de problemas con un retorno lento de la inversión $(5-7,12)$ 


\section{Conclusiones}

El estudio del COVID-19 constituye un tema de actualidad debido a la amplia investigación asociada y a la rápida propagación del virus; recientemente fue declarado como pandemia por la OMS. Las cifras máximas actuales de mortalidad asociadas al virus rondan el $3 \%$ y su presentación severa es más prevalente en pacientes con enfermedades crónicas subyacentes.

El diagnóstico eficaz mediante la evolución clínica, pruebas de gabinete y detección de ARN viral por RT-PCR facilitan el manejo oportuno con medidas de soporte adecuadas, y concomitantemente la prevención corresponde un pilar fundamental dentro del manejo de los casos de coronavirus. Aún no existe un protocolo de tratamiento en las guías actuales para COVID-19 y el desarrollo de vacunas necesita progresar a fases más avanzadas.

\section{Declaración de conflicto de intereses}

Las autoras declaran que no existió ningún conflicto de interés en el presente reporte.

\section{Referencias biliográficas}

1. Chen J. Pathogenicity and Transmissibility of 2019-nCoV-A Quick Overview and Comparison with Other Emerging Viruses. Microbes and Infection. 2020;2019-2021.

2. Cheng ZJ, Shan J. 2019 Novel Coronavirus: Where We are and What We Know. Infection. 2020.

3. Chang D, Xu H, Rebaza A, Sharma L, Dela Cruz CS. Protecting health-care workers from subclinical coronavirus infection. The Lancet: Respiratory Medicine. 2020;2600(20):2001468.

4. Han W, Quan B, Guo Y, Zhang J, Feng G,
5. Wu Q, et al. Letter to the editor:The course of clinical diagnosis and treatment of a case infected with coronavirus disease 2019. Journal of Medical Virology. 2020;1-2.

6. Wang C, Horby PW, Hayden FG, Gao GF. A novel coronavirus outbreak of global health concern. The Lancet. 2020;395:15-8.

7. Liu Y, Liao C, Chang C, Chou C-C, Lin Y-R. Correspondence: Multidrug-Resistant Tuberculosis. New England Journal of Medicine. 2020;4-6.

8. Yu F, Du L, Ojcius DM, Pan C, Jiang S. Measures for diagnosing and treating infections by a novel coronavirus responsible for a pneumonia outbreak originating in Wuhan, China. Microbes and Infection. 2020;1-6.

9. Huang C, Wang Y, Li X, Ren L, Zhao J, Hu $\mathrm{Y}$, et al. Clinical features of patients infected with 2019 novel coronavirus in Wuhan, China. The Lancet . 2020;497-506.

10. Jiang S, Shi Z-L. The First Disease $\mathrm{X}$ is Caused by a Highly Transmissible Acute Respiratory Syndrome Coronavirus. Virologica Sinica. 2020;12250:5-7.

11. Holshue ML, DeBolt C, Lindquist S, Lofy KH, Wiesman J, Bruce H, et al. First Case of 2019 Novel Coronavirus in the United States. New England Journal of Medicine . 2020;1-9.

12. Zhu N, Zhang D, Wang W, Li X, Yang B, Song J, et al. A Novel Coronavirus from Patients with Pneumonia in China, 2019. New England Journal of Medicine 2020;1-7.

13. Wang D, Hu B, Hu C, Zhu F, Liu X, Zhang J, et al. Clinical Characteristics of 138 Hospitalized Patients With 2019 Novel Coronavirus-Infected Pneumonia in Wuhan, China. JAMA. 2020;1-9. 
14. Chan JFW, Yuan S, Kok KH, To KKW, Chu

15. H, Yang J, et al. A familial cluster of pneumonia associated with the 2019 novel coronavirus indicating person-to-person transmission: a study of a family cluster. The Lancet. 2020;395(10223):514-23.

16. World Health Organization. Situation report - 53: Coronavirus disease 2019 (COVID-19) [Internet]. 2020. p. 1-10. Available from: https://www.who.int/docs/default-source/cor onaviruse/situation-reports/20200313-sitrep -53-covid-19.pdf?sfvrsn=adb3f72_2

17. Munster V, Koopmans M, Doremalen N, Van Riel D, Wit E. A Novel Coronavirus Emerging in China - Key Questions for Impact Assessment. New England Journal of Medicine . 2020;1-3.

18. Zhang J, Zhout L, Wang W, Chen X. Correspondence: Therapeutic and triage strategies for 2019 novel coronavirus. The Lancet: Respiratory Medicine.

2020;2600(20):2019-20.

19. Chen N, Zhou M, Dong X, Qu J, Gong F, Han $\mathrm{Y}$, et al. Epidemiological and clinical characteristics of 99 cases of 2019 novel coronavirus pneumonia in Wuhan, China: a descriptive study. The Lancet. 2020;395(10223):507-13.

20. World Health Organization. Interim guidance: Clinical management of severe acute respiratory infection when novel coronavirus $(\mathrm{nCoV})$ infection is suspected. [Internet]. 2020. p. 12. Available from: https://www.who.int/docs/default-source/cor onaviruse/clinical-management-of-novel-cov .pdf

21. Phan T. Novel coronavirus: From discovery to clinical diagnostics. Infection, Genetics and Evolution. 2020;79:104211. Zhang L, Liu Y. Potential Interventions for Novel Coronavirus in China: A Systemic
22. Review. Journal of Medical Virology. 2020; $1-36$.

23. Paraskevis D, Kostaki EG, Magiorkinis G, Panayiotakopoulos G, Sourvinos G, Tsiodras S. Full-genome evolutionary analysis of the novel corona virus (2019-nCoV) rejects the hypothesis of emergence as a result of a recent recombination event. Infection, Genetics and Evolution. 2020;79:104212.

24. Pongpirul WA, Pongpirul K, Ratnarathon AC, Prasithsirikul W. Correspondence: Journey of a Thai Taxi Driver and Novel Coronavirus. New England Journal of Medicine. 2020;1.

25. Bastola A, Sah R, Rodriguez-morales AJ, Lal $\mathrm{BK}$, Jha R, Ojha HC, et al. Correspondence: coronavirus case in. The Lancet Infectious Diseases . 2020;3099(20):2019-20.

26. Rothe C, Schunk M, Sothmann P, Bretzel G, Froeschl G, Wallrauch C, et al.

Transmission of 2019-nCoV Infection from an Asymptomatic Contact in Germany. New England Journal of Medicine. 2020;2019-20.

27. Li Q, Guan X, Wu P, Wang X, Zhou L, Tong $\mathrm{Y}$, et al. Early Transmission Dynamics in Wuhan, China, of Novel Coronavirus-Infected Pneumonia. New England Journal of Medicine. 2020;1-9.

28. Phan LT, Nguyen T V., Luong QC, Nguyen T V., Nguyen HT, Le HQ, et al. Importation and Human-to-Human Transmission of a Novel Coronavirus in Vietnam. New England Journal of Medicine. 2020.

29. The Lancet Journal. Emerging understandings of $2019-\mathrm{nCoV}$. The Lancet. 2020;395(10221):311.

30. Hoehl S, Rabenau H, Berger A, Kortenbusch M, Cinatl J, Bokjova D. Correspondece: 
31. Evidence of SARS-CoV-2 Infection in Returning Travelers from Wuhan, China. New England Journal of Medicine.

2020;1-3.

32. Lipsitch M, Phil D, Swerdlow DL, Finelli L. Perspective: Defining the Epidemiology of Covid-19 - Studies Needed. New England Journal of Medicine. 2020;1-3.

33. Xu X, Yu C, Zhang L, Luo L, Liu J. Letter to editor: Imaging features of 2019 novel coronavirus pneumonia. European Journal of Nuclear Medicine and Molecular Imaging . 2020;1-2.

34. Wang M, Cao R, Zhang L, Yang X, Liu J, Xu $\mathrm{M}$, et al. Remdesivir and chloroquine effectively inhibit the recently emerged novel coronavirus (2019-nCoV) in vitro. Cell Res . 2020;(January):2019-21.

35. Gao J, Tian Z, Yang X. Breakthrough: Chloroquine phosphate has shown apparent efficacy in treatment of COVID-19 associated pneumonia in clinical studies.

Bioscience Trends. 2020;1-2. 